\title{
Sweet taste signaling and the formation of memories of energy sources
}

\section{Ivan E. de Araujo*}

The John B. Pierce Laboratory, Department of Psychiatry, Yale University School of Medicine, New Haven, CT, USA

Edited by:

Edmund Rolls, University of

Oxford, UK

\section{Reviewed by:}

Milagros Gallo, University of

Granada, Spain

Edmund Rolls, University of

Oxford, UK

\section{*Correspondence:}

Ivan E. de Araujo, The John B.

Pierce Laboratory, Department of

Psychiatry, Yale University School of

Medicine, 290 Congress Avenue,

New Haven, CT 06519, USA.

e-mail: iaraujo@jbpierce.org
The last decade witnessed remarkable advances in our knowledge of the gustatory system. Application of molecular biology techniques not only determined the identity of the membrane receptors and downstream effectors that mediate sweetness, but also uncovered the overall logic of gustatory coding in the periphery. However, while the ability to taste sweet may offer the obvious advantage of eliciting rapid and robust intake of sugars, a number of recent studies demonstrate that sweetness is neither necessary nor sufficient for the formation of long-lasting preferences for stimuli associated with sugar intake. Furthermore, uncoupling sweet taste from ensuing energy utilization may disrupt body weight control. This minireview examines recent experiments performed in both rodents and Drosophila revealing the taste-independent rewarding properties of metabolizable sugars. Taken together, these experiments demonstrate the reinforcing actions of sugars in the absence of sweet taste signaling and point to a critical role played by dopamine systems in translating metabolic sensing into behavioral action. From a mechanistic viewpoint, current evidence favors the concept that gastrointestinal and post-absorptive signals contribute in parallel to sweet-independent sugar acceptance and dopamine release.

Keywords: carbohydrates, dopamine, glucose oxidation, nutrient selection, reward, striatum, sweet taste, TRPM5

\section{INTRODUCTION}

Glucose-containing carbohydrates, the ingestion of which are critical for most forms of animal life, reliably elicit the highly pleasurable sensation of sweetness. Such mechanism allows the brain to rapidly trigger acceptance responses upon recognizing the presence of nutritive carbohydrates in the oral cavity. Given the consistency of this behavioral response, one would hypothesize that sweet taste is both necessary and sufficient for the appropriate control of sugar intake. However, mounting evidence from both mammals and insects now favors the possibility that long-term food choices depend primarily on the detection of the energy content of the food sources, without requiring the stimulation of sweet taste effectors. This minireview concentrates on describing recent studies in both rodents and Drosophila revealing that the formation of long-term sugar acceptance and preference requires the activation of energy-sensing pathways.

\section{THE GUSTATORY SYSTEM}

Recent reviews have covered in depth the anatomy of both the peripheral and central gustatory pathways (Carleton et al., 2010; Kinnamon, 2011), which we will mention only briefly. The peripheral gustatory system consists of the neural-epithelial machinery linking the sensory epithelial cells in the oral cavity to the first gustatory relay center in the brain. G-proteincoupled receptors (GPCRs) expressed on the apical end of taste receptor cells (TRCs) function as the receptors for sweet, some L-amino acids, and bitter tastants while ion channels of the transient receptor potential are thought to mediate sour and salty tastes (Chandrashekar et al., 2006; Roper, 2007). It is noticeable that taste receptor expression has been detected at other organs including the gastrointestinal tract (Margolskee et al., 2007; Hass et al., 2010), pancreas (Nakagawa et al., 2009), and brain (Ren et al., 2009), although extra-oral physiological functions remain to be determined. On the tongue, sweet taste is specifically mediated by the taste genes Tas1r2 and Tas1r3, whose T1R2 and T1R3 products assemble to form the heterodimeric sweet receptor T1R2/T1R3 (Chandrashekar et al., 2006). T1R2/T1R3 activation is effected via the downstream signals phospholipase PLC $\beta 2$ and TRPM5, a non-selective ionic taste channel, the deletion of either inducing severe impairment in-if not taste-blindness for-sweet, umami, and bitter transduction (Zhang et al., 2003). Sweet tastant-induced TRC depolarizationmediated by TRPM5-produces the release of chemical messages onto cranial nerve afferents innervating the basolateral aspect of TRCs. The cell bodies of taste-responsive cranial nerve ganglia synapse into the rostral division of the nucleus tractus solitarius (rNTS) in the medulla (Hamilton and Norgren, 1984), which in turn projects, in rodents, ipsilaterally to the parabrachial nucleus (PBN, Norgren and Leonard, 1971). From PBN two pathways ascend in parallel to the forebrain: a "dorsal" pathway projecting to parvicellular areas of the ventroposterior medial nucleus of the thalamus (whose afferents define anatomically the primary gustatory cortex in the insula), and one ventral pathway projecting to the amygdala, lateral hypothalamus, and the bed nucleus of the stria terminalis (Norgren, 1976). Noticeably NTS taste projections seem to ascend directly to thalamus, therefore bypassing the PBN (Small and Scott, 2009). 


\section{SWEET TASTE AND BRAIN DOPAMINE RELEASE}

Robust attraction toward sweet tastants is pervasive across most species. Animals will avidly consume sweet solutions even when required to learn complex operant behaviors (Kare, 1971). Innate attraction to sweetness is presumed in humans given the stereotyped oral/facial reactions observed in children upon their first exposure to sugary solutions (Ganchrow et al., 1983). However, recent evidence reveals that early childhood flavor exposures rather than innateness per se mold long-term food preferences (Beauchamp and Mennella, 2009; Ventura and Mennella, 2011). It does, therefore, seem logical to presume that neural pathways must exist that link peripheral sweet receptors to brain rewardrelated circuits (McCaughey, 2008). Among such circuits we must stress the brain cells producing the reward-related monoaminergic transmitter dopamine. Dopamine is a major regulator of sweet sugar intake. In fact, dopamine receptor antagonism decreases the attraction toward sweet-tasting nutrients given that animals pretreated with either D1- or D2-type dopamine receptor antagonists approach high concentrations of sucrose solutions as if they had been diluted (Xenakis and Sclafani, 1981; Wise, 2006). Conversely, palatable foods elevate extracellular dopamine concentrations in the nucleus accumbens (NAcc) of the ventral striatum (Hernandez and Hoebel, 1988), a brain region critical for the expression of normal feeding behaviors (Kelley et al., 2005). In humans, striatal dopamine release reflects the perceived pleasantness of a meal (Small et al., 2003). Sweet-elicited stimulation of the central dopamine systems occurs upon intra-oral stimulation but does not require intestinal sugar absorption (as demonstrated in "sham-feeding" preparations combined with microdialysis measurements, Hajnal et al., 2004), as effect found to depend on the integrity of the "ventral" taste pathway (Norgren and Hajnal, 2005). It does, therefore, appear that sensing sweetness per se would account for the high acceptance associated with sugar intake.

\section{SWEETNESS-INDEPENDENT ATTRACTION TO SUGARS}

While sweet sensation is a powerful drive of feeding behavior, it remains to be proven that animals rely entirely on the orosensory properties of sugars to evaluate energy sources. In fact, it has been long established that approach or satiation responses to a given flavor can be conditioned by postingestive consequences (Sclafani and Xenakis, 1984; Booth, 1985; Rolls, 2005). For instance, in flavor-nutrient conditioning experiments, gut infusions of a given nutrient or control solution are conditionally linked to the oral intake of a distinct flavor, usually represented by odorant solutions that had been artificially sweetened (Sclafani and Xenakis, 1984; Booth, 1985). These experiments show very clearly that rodents (Booth, 1985; Sclafani, 2001) and humans (Hellstrom et al., 2004) will develop strong preferences for flavors that had been paired to infusions of nutrients compared to control infusions, with a bias toward glucose-containing sugars over other isocaloric nutrients (Ackroff and Sclafani, 2006).

Would animals develop preferences for sugars even when not paired to distinct sweet flavors? To assess this possibility the author designed a conditioning protocol where mice are allowed to form memories of sipper locations that had been previously associated with the oral delivery of sugars, water, or non-caloric sweeteners (de Araujo et al., 2008). The study involved employing wild-type as well as knockout mice lacking functional TRPM5 channels (Zhang et al., 2003). As was mentioned above, the TRPM5 ion channel is expressed in TRCs (Perez et al., 2002) and is required for sweet taste signaling (Zhang et al., 2003). Accordingly, it was hypothesized that sweet-blind Trpm 5 knockout mice would be able to form robust preferences for those spouts previously associated with the oral presentation of sucrose solutions as long as these animals were allowed sufficient time to detect the solutions' postingestive effects. This was accomplished by first determining the initial side-preferences using a series of pre-conditioning two-bottle water tests, followed by exposing animals to $30 \mathrm{~min}-$ long conditioning sessions where either water (assigned to the same side of initial bias) or sucrose (assigned to the opposite side) were consumed freely while access to the other sipper was blocked.

Results from this experiment demonstrated that, unlike during short-term exposure, during the $30 \mathrm{~min}$ conditioning sessions both wild-type and knockout animals consumed significantly larger amounts of sucrose compared to water. In addition, during post-conditioning two-bottle tests, both wildtype and knockout animals reversed their initial side-preference biases by consuming significantly more water from those sippers that during conditioning sessions had been associated with sucrose. Therefore, oral stimulation with sweetness or otherwise distinct flavors was not required to induce strong biases toward consuming nutritive sucrose. These effects were in fact dependent on the energy content, rather than sweetness per se, associated with sucrose since when the same experiments were performed using the non-caloric sucrose-derived sweetener sucralose instead of sucrose, only wild-type animals consumed more sucralose than water during the conditioning sessions.

However, and critical to our argument in this review, during the two-bottle post-conditioning water sessions, neither knockout nor wild-type mice showed preferences for sippers associated with the delivery of sucralose. Overall, these results provide evidence in favor of the hypothesis that sweetness is neither necessary nor sufficient to induce long-term sugar preferences if unaccompanied by detectable physiological effects.

\section{POSTINGESTIVE SIGNALS AND BRAIN DOPAMINE RELEASE}

Given the above, it would be natural to conclude that sweetness may not be required for dopamine to be released during sucrose intake. In fact, microdialysis measurements revealed on one hand that the non-caloric sweetener sucralose produced significantly higher increases in NAcc dopamine levels in wild-type compared to TRPM5 knockout animals (de Araujo et al., 2008). These results are consistent with the ability of sweetness per se to stimulate dopamine release in NAcc (Hajnal et al., 2004). Now, when the same comparison was performed using sucrose, no differences were found between NAcc dopamine levels in wild-type and TRPM5 knockout mice. In conclusion, while sweet taste stimulation without caloric content only produced significant increases in accumbal dopamine levels in wild-type, caloric sucrose evoked 
the same levels of dopamine increase in both wild-type and sweet-blind mice.

The above is also consistent with the fact that rats treated with local infusions in NAcc with a D1-receptor antagonist display dose-dependent reductions in intake of a flavor paired with intra-gastric infusions of glucose (Touzani et al., 2008). Interestingly, the effect of dopamine signaling antagonism on post-conditioning preferences tests was less compelling (Touzani et al., 2008). In any event, these results demonstrate that D1-like receptors in the NAcc are required for the acquisition of glucose-conditioned flavor preferences. Finally it must be noted that in addition to striatum other brain regions including the amygdala, lateral hypothalamus, and medial prefrontal cortex mediate postingestive influences on behavior (Sclafani et al., 2011), although it is intriguing to note that all those are densely targeted by dopaminergic afferents.

\section{SWEETNESS-INDEPENDENT ATTRACTION FOR SUGARS IN DROSOPHILA}

The attraction to sugars in the absence of sweetness or distinct flavors does not seem to be limited to vertebrates. Two very interesting recent studies independently report that flies not only survive by feeding on a tasteless metabolizable sugar, but will form odor-sugar memories only when sugar cues provide metabolic benefit. Their strategy was based on the elegant idea of comparing the results obtained from attempting to condition behavioral approach to a sweet, non-metabolizable sugar against those obtained from conditioning approach to a non-sweet, nutritional sugar (reviewed in Wright 2011). Burke and Waddell (2011) have shown that flies will not form lasting memories for odors that had been previously associated with non-metabolizable sugars such as arabinose. In fact, when these authors added the nonsweet (to flies) alcohol sugar D-sorbitol to arabinose, memory retrieval was as efficient as when odors were paired to sucrose.

The ability of flies to recognize the nutritional value of sugars independently of taste was also shown by Fujita and Tanimura (2011). These authors have also shown that flies can form associations between odors and arabinose only if D-sorbitol is added. In addition, flies were able to maintain normal physiological functions when given D-sorbitol as the only nutrient available (Fujita and Tanimura, 2011). These authors have also shown that neural mechanisms must be involved in the learning processes described above, since null mutants of the synapsin gene $s y n^{97}$, which encodes a protein necessary for synaptic function, showed significant reductions in the ability to associate arbitrary odors with tasteless nutritive sugars (Fujita and Tanimura, 2011).

It is intriguing to note that in Drosophila, as in mammals, dopaminergic pathways play a role in regulating behavioral responses to rewarding stimuli such as cocaine, nicotine, and ethanol (Bainton et al., 2000). In addition, blocking transmission in dopaminergic neurons abolishes the expression of conditioned preferences for ethanol-associated cues (Kaun et al., 2011). It is therefore plausible to hypothesize that dopamine may mediate the ability to sense the nutritional value of sugars, including tasteless sorbitol, in flies as it does in mammals.

\section{UNCOUPLING SWEET TASTE FROM ENERGY UTILIZATION}

Another interesting aspect associated with the relationship between sweet taste and sugar metabolism relates to the fact that the usage of non-caloric sweeteners may disrupt the predictive relationship between sweetness and energy intake. Swithers, Davidson, and colleagues developed an experimental rodent model to study the role of sweet taste as a predictor of energy intake (see e.g., Swithers and Davidson, 2008; Swithers et al., 2009). Overall, the results reveal that intake of foods (or fluids) containing non-nutritive sweeteners, when compared to the intake of glucose, leads to significant weight gain, increased fat deposition, and impaired ability to caloric compensation. Overall, these results suggest that consumption of saccharin or other non-caloric sweeteners may decrease the ability of the organism to upregulate energy utilization, a physiological response that usually follows sugar ingestion (Swithers et al., 2009).

\section{ON THE IDENTITY OF THE POSTINGESTIVE REINFORCING SIGNAL}

A critical question that remains to be resolved regards the identity of the taste-independent reinforcement signal. Generally speaking, candidate signals could be classified into two major groups, according to whether they are generated during either pre- or post-absorptive phases of food intake. The former group includes, broadly speaking, those signals occurring previous to nutrient delivery into the bloodstream but simultaneous to the arrival of nutrients to the gut. The latter group on the other hand refers to those events occurring after nutrients reach the bloodstream, and non-exclusively includes a variety of signals such as fuel utilization metabolites and changes in plasma hormonal levels.

Experiments based on flavor-nutrient conditioning paradigms indicate that pre-absorptive signals may mediate the ability to form associations between orally delivered flavors and intragastrically delivered sugars. In fact, no flavor preference learning was obtained when flavor intake was paired with portal infusions of glucose (Ackroff et al., 2010). It is interesting to note that flavor-nutrient conditioning is robustly achieved even when infusions bypass the stomach and are delivered directly to the small intestine (Ackroff et al., 2010). These results are supported by the fact that abdominal vagotomy does not interfere with flavor preferences conditioned by glucose-containing sugars (Sclafani and Lucas, 1996). Altogether, the above allowed Sclafani and colleagues to infer that a currently unknown glucose sensor expressed in the intestine mediates flavor-nutrient conditioning to glucose-containing sugars (Ackroff et al., 2010).

Physiological signals generated post-absorption also seem to regulate sweet-independent attraction to sugars. We have recently assessed in our laboratory the potential role of metabolic signals in taste-independent nutrient selection by comparing the behavioral responses to glucose and weakly gluconeogenic L-amino acids in wild-type and Trpm5 knockout mice. Briefly, Trpm5 knockout mice, despite displaying insensitivity to the tastes of both glucose and L-serine during short-term (10 $\mathrm{min})$ tests, did form strong preferences for glucose-associated sippers during conditioning sessions, as well as ingested significant larger amounts of glucose during longer-term sessions (Ren et al., 2010). These results were confirmed by indirect calorimetry 
measurements, which demonstrated that higher intake levels of glucose were closely associated with glucose oxidation levels, in such a way that respiratory quotient measures functioned as highly efficient predictors of intake, even more so than blood glucose levels. This finding points to a role for post-absorptive nutrient utilization in postingestive reinforcement.
Consistent with the above, glucose utilization rates were also found to act as one powerful regulator of dopamine release. In fact, we have shown in the same study that an intravenous infusion of the anti-metabolic glucose analog, 2-deoxy-D-glucose (henceforth "2-DG") resulted in significant decreases in extracellular dopamine levels. In addition, such inhibitory effects of

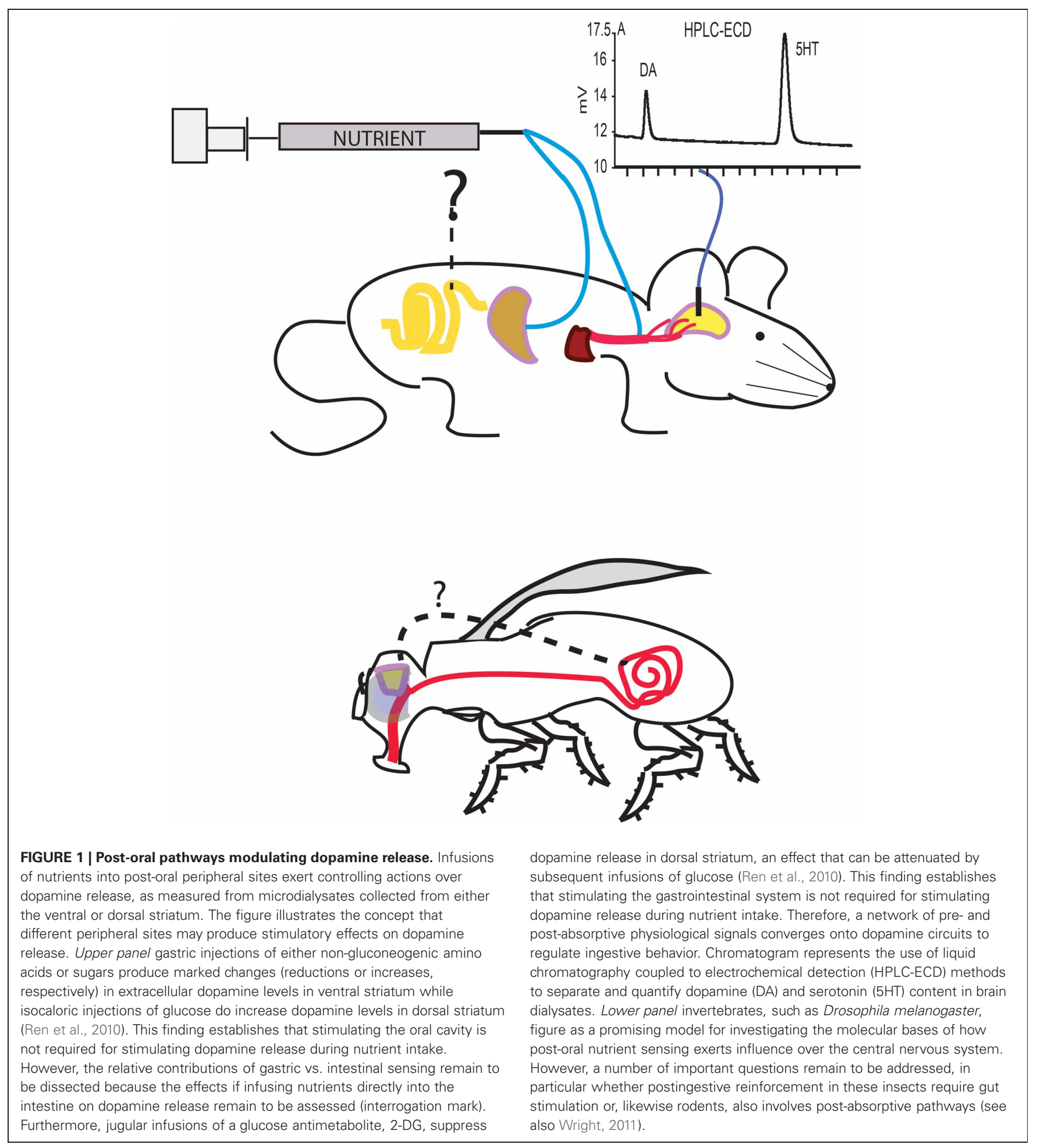


2-DG on dopamine release were attenuated, or almost reversed, by a subsequent intravenous glucose infusion that counteracted 2-DG effects and contributed to restore normal glucose oxidation rates (Ren et al., 2010). Finally, but always consistent with a role for glucose utilization in mediating sweet-independent sugar reinforcement, we have also found that after 2-DG injections Trpm 5 knockout mice produced significantly higher numbers of licks to glucose compared to after saline injections. Thus, within 30 min of 2-DG administration, glucose becomes more attractive if contributing to reinstate glucose oxidation levels.

Altogether, the results above indicate that both intestinal and metabolic factors simultaneously convey physiological signals that affect the central nervous system. It is important to note that these two pathways are not necessarily mutually exclusive. In fact, they are more likely to cooperate to control both acceptance and preference for environmental stimuli associated with sugars. For example, it is possible that metabolic signals are important for regulating overall sugar intake levels (as demonstrated by the 2-DG experiments performed by Ren et al., 2010) whereas preferences for flavors associated with intra-gastric glucose depend on vagus-independent intestinal signals (Ackroff et al., 2010). Alternatively, the intestine may trigger the release of incretin factors that ultimately may enhance insulin release, and, therefore, glucose uptake and utilization. Future research must determine the signaling pathways that

\section{REFERENCES}

Ackroff, K., and Sclafani, A. (2006). Energy density and macronutrient composition determine flavor preference conditioned by intragastric infusions of mixed diets. Physiol. Behav. 89, 250-260.

Ackroff, K., Yiin, Y. M., and Sclafani, A. (2010). Post-oral infusion sites that support glucose-conditioned flavor preferences in rats. Physiol. Behav. 99, 402-411.

Bainton, R. J., Tsai, L. T., Singh, C. M., Moore, M. S., Neckameyer, W. S., and Heberlein, U. (2000). Dopamine modulates acute responses to cocaine, nicotine and ethanol in Drosophila. Curr. Biol. 10, 187-194.

Beauchamp, G. K., and Mennella, J. A. (2009). Early flavor learning and its impact on later feeding behavior. J. Pediatr. Gastroenterol. Nutr. 48(Suppl. 1), S25-S30.

Booth, D. A. (1985). Food-conditioned eating preferences and aversions with interoceptive elements: conditioned appetites and satieties. Ann. N. Y. Acad. Sci. 443, 22-41.

Burke, C. J., and Waddell, S. (2011). Remembering nutrient quality of sugar in Drosophila. Curr. Biol. 21, 746-750.

Carleton, A., Accolla, R., and Simon, S. A. (2010). Coding in the mammalian gustatory system. Trends Neurosci. 33, 326-334.
Chandrashekar, J., Hoon, M. A., Ryba, N. J., and Zuker, C. S. (2006). The receptors and cells for mammalian taste. Nature 444, 288-294.

de Araujo, I. E., Oliveira-Maia, A. J., Sotnikova, T. D., Gainetdinov, R. R., Caron, M. G., Nicolelis, M. A., and Simon, S. A. (2008). Food reward in the absence of taste receptor signaling. Neuron 57, 930-941.

Fujita, M., and Tanimura, T. (2011). Drosophila evaluates and learns the nutritional value of sugars. Curr. Biol. 21, 751-755.

Ganchrow, J. R., Steiner, J. E., and Daher, M. (1983). Neonatal facial expressions in response to different qualities and intensities of gustatory stimuli. Infant Behav. Dev. 6, 473-484.

Hajnal, A., Smith, G. P., and Norgren, R. (2004). Oral sucrose stimulation increases accumbens dopamine in the rat. Am. J. Physiol. Regul. Integr. Comp. Physiol. 286, R31-R37.

Hamilton, R. B., and Norgren, R. (1984). Central projections of gustatory nerves in the rat. J. Comp. Neurol. 222, 560-577.

Hass, N., Schwarzenbacher, K., and Breer, H. (2010). T1R3 is expressed in brush cells and ghrelin-producing cells of murine stomach. Cell Tissue Res. 339, 493-504.

allow brain dopamine systems to sense the energy of sugars without requiring inputs form the oral cavity. One interesting hypothesis consists in the possibility that dopamine neurons have the ability to sense the internal energy levels of the cell, modulating transmitter release accordingly, possibly via cellular sensors such as AMPK. This feature would place dopamine neurons among the brain's glucosensors, as hypothesized previously (Levin, 2000).

\section{CONCLUSION}

We have reviewed evidence that favors the conclusion that sweet taste signaling is neither necessary nor sufficient to allow for the formation of lasting memories or preferences for sugar-associated stimuli. Furthermore, brain circuits, particularly dopaminergic systems, show marked sensitivity to the energy content of sugars independently of oral stimulation. Current evidence points to the possibility that the brain monitors both gastrointestinal signals and energy utilization rates to control sugar intake independently of the sense of sweet. Future research must determine the physiological pathways allowing the gastrointestinal system and intracellular energy sensors to control dopamine release during sugar intake (See Figure 1).

\section{ACKNOWLEDGMENT}

This work was supported by NIH grant DC009997 to IEA.

Hellstrom, P. M., Geliebter, A., Naslund, E., Schmidt, P. T., Yahav, E. K., Hashim, S. A., and Yeomans, M. R. (2004). Peripheral and central signals in the control of eating in normal, obese and bingeeating human subjects. Br. J. Nutr. 92(Suppl. 1), S47-S57.

Hernandez, L., and Hoebel, B. G. (1988). Food reward and cocaine increase extracellular dopamine in the nucleus accumbens as measured by microdialysis. Life Sci. 42 , 1705-1712.

Kare, M. R. (1971). "Comparative study of taste," in Handbook of Sensory Physiology, ed L. M. Beidler (Berlin: Springer-Verlag), 278-292.

Kaun, K. R., Azanchi, R., Maung, Z., Hirsh, J., and Heberlein, U. (2011). A Drosophila model for alcohol reward. Nat. Neurosci. 14, 612-619.

Kelley, A. E., Schiltz, C. A., and Landry, C. F. (2005). Neural systems recruited by drug- and food-related cues: studies of gene activation in corticolimbic regions. Physiol. Behav. 86, 11-14.

Kinnamon, S. C. (2011). Taste receptor signalling - from tongues to lungs. Acta Physiol. (Oxf) 203, 1-11.

Levin, B. E. (2000). Glucose-regulated dopamine release from substantia nigra neurons. Brain Res. 874 , 158-164.
Margolskee, R. F., Dyer, J., Kokrashvili, Z., Salmon, K. S., Ilegems, E., Daly, K., Maillet, E. L., Ninomiya, Y., Mosinger, B., and Shirazi-Beechey, S. P. (2007). T1R3 and gustducin in gut sense sugars to regulate expression of $\mathrm{Na}+$-glucose cotransporter 1. Proc. Natl. Acad. Sci. U.S.A. 104, 15075-15080.

McCaughey, S. A. (2008). The taste of sugars. Neurosci. Biobehav. Rev. 32, 1024-1043.

Nakagawa, Y., Nagasawa, M., Yamada, S., Hara, A., Mogami, H., Nikolaev, V. O., Lohse, M. J., Shigemura, N., Ninomiya, Y., and Kojima, I. (2009). Sweet taste receptor expressed in pancreatic beta-cells activates the calcium and cyclic AMP signaling systems and stimulates insulin secretion. PLoS One, 4, e5106. doi: 10.1371/journal.pone.0005106

Norgren, R. (1976). Taste pathways to hypothalamus and amygdala. $J$. Comp. Neurol. 166, 17-30.

Norgren, R., and Hajnal, A. (2005). Taste pathways that mediate accumbens dopamine release by sapid sucrose. Physiol. Behav. 84, 363-369.

Norgren, R., and Leonard, C. M. (1971). Taste pathways in rat brainstem. Science 173, 1136-1139.

Perez, C. A., Huang, L., Rong, M., Kozak, J. A., Preuss, A. K., Zhang, H., Max, M., and Margolskee, R. F. (2002). A transient receptor 
potential channel expressed in taste receptor cells. Nat. Neurosci. 5, 1169-1176.

Ren, X., Ferreira, J. G., Zhou, L., Shammah-Lagnado, S. J., Yeckel, C. W., and de Araujo, I. E. (2010). Nutrient selection in the absence of taste receptor signaling. J. Neurosci. 30, 8012-8023.

Ren, X., Zhou, L., Terwilliger, R., Newton, S. S., and de Araujo, I. E. (2009). Sweet taste signaling functions as a hypothalamic glucose sensor. Front. Integr. Neurosci. 3, 12. doi:10.3389/neuro.07.012.2009

Rolls, E. T. (2005). Emotion Explained. Oxford: Oxford University Press.

Roper, S. (2007). Signal transduction and information processing in mammalian taste buds. Pflugers Arch. 454, 759-776.

Sclafani, A. (2001). Post-ingestive positive controls of ingestive behavior. Appetite 36, 79-83.

Sclafani, A., and Lucas, F. (1996). Abdominal vagotomy does not block carbohydrate-conditioned flavor preferences in rats. Physiol. Behav. 60, 447-453.
Sclafani, A., Touzani, K., and Bodnar, R. J. (2011). Dopamine and learned food preferences. Physiol. Behav. 104, 64-68.

Sclafani, A., and Xenakis, S. (1984). Sucrose and polysaccharide induced-obesity in the rat. Physiol. Behav. 32, 169-174.

Small, D. M., Jones-Gotman, M., and Dagher, A. (2003). Feeding-induced dopamine release in dorsal striatum correlates with meal pleasantness ratings in healthy human volunteers. Neuroimage 19, 1709-1715.

Small, D. M., and Scott, T. R. (2009). Symposium overview: what happens to the pontine processing? Repercussions of interspecies differences in pontine taste representation for tasting and feeding. Ann. N. Y. Acad. Sci. 1170, 343-346.

Swithers, S. E., Baker, C. R., and Davidson, T. L. (2009). General and persistent effects of high-intensity sweeteners on body weight gain and caloric compensation in rats. Behav. Neurosci. 123, 772-780.

Swithers, S. E., and Davidson, T. L. (2008). A role for sweet taste: calorie predictive relations in energy regulation by rats. Behav. Neurosci. 122 161-173.

Touzani, K., Bodnar, R., and Sclafani, A. (2008). Activation of dopamine D1-like receptors in nucleus accumbens is critical for the acquisition, but not the expression, of nutrientconditioned flavor preferences in rats. Eur. J. Neurosci. 27, 1525-1533. Ventura, A. K., and Mennella, J. A. (2011). Innate and learned preferences for sweet taste during childhood. Curr. Opin. Clin. Nutr. Metab. Care 14, 379-384.

Wise, R. A. (2006). Role of brain dopamine in food reward and reinforcement. Philos. Trans. $R$ Soc. Lond. B Biol. Sci. 361, 1149-1158.

Wright, G. A. (2011). Appetitive learning: memories need calories. Curr. Biol. 21, R301-R302.

Xenakis, S., and Sclafani, A. (1981). The effects of pimozide on the consumption of a palatable saccharin-glucose solution in the rat. Pharmacol. Biochem. Behav. 15, 435-442.

Zhang, Y., Hoon, M. A., Chandrashekar, J., Mueller, K. L., Cook,
B. W. D., Zucker, C. S., and Ryba, N. J. (2003). Coding of sweet, bitter, and umami tastes: different receptor cells sharing similar signaling pathways. Cell 112, 293-301.

Conflict of Interest Statement: The authors declare that the research was conducted in the absence of any commercial or financial relationships that could be construed as a potential conflict of interest.

Received: 10 September 2011; accepted: 18 November 2011; published online: 29 November 2011.

Citation: de Araujo IE (2011) Sweet taste signaling and the formation of memories of energy sources. Front. Syst. Neurosci. 5:99. doi: 10.3389/fnsys.2011.00099 Copyright (c) 2011 de Araujo. This is an open-access article distributed under the terms of the Creative Commons Attribution Non Commercial License, which permits non-commercial use, distribution, and reproduction in other forums, provided the original authors and source are credited. 